Materialeinsatz und Industrial Ecology

\title{
Ressourceneffizienz als Chance für die Industrial Ecology
}

\author{
Hinter dem Begriff der Ressourceneffizienz \\ verbergen sich verschiedene Zielsetzungen der \\ Nachhaltigkeit, aber auch der Wirtschaft und \\ der Politik. Er bietet viele Interpretationsmöglich- \\ keiten und Handlungsansätze. Eine neue \\ Richtlinie versucht nun, begrifflich und metho- \\ disch einen Rahmen vorzugeben. \\ Von Mario Schmidt
}

$\mathrm{N}$ iemand wird bestreiten, dass Ressourcen wichtig sind und die Grundlage unserer modernen Gesellschaft darstellen. Effizienz im Mitteleinsatz ist fast eine Doktrin unserer Zeit. Nur wenige wehren sich aus kulturphilosophischen Gründen; einige warnen vor Reboundeffekten. Aber die Wirtschaft kann mit dem Begriff mehr anfangen als mit dem inzwischen abgegriffenen Wort der Nachhaltigkeit.

Dabei gibt es bei der Ressourceneffizienz einen vergleichbaren Interpretationsspielraum, der von den Akteuren - bewusst oder unbewusst - meistens aber positiv im Kontext ihres Handlungsumfeldes gefüllt wird. Das führt dazu, dass unter Ressourceneffizienz ganz unterschiedliche Dinge zusammengefasst werden. Der kleinste gemeinsame Nenner ist vielleicht, dass man Verschwendung vermeiden will. Aber Verschwendung von was?

Am einfachsten ist noch die Entscheidung, auf welche Ressourcen man sich konzentriert. Gemeint sind nicht die Human Resources oder der Kapitaleinsatz der Unternehmen, sondern die natürlichen Ressourcen. In der aktuellen Diskussion werden die energetischen Ressourcen meistens ausgeklammert, da sie zentraler Bestandteil eines parallelen Handlungsfeldes, nämlich der Energiewende, sind. Im Ressourceneffizienzprogramm der Bundesregierung wird zum Beispiel auf die stoffliche Nutzung von abiotischen, teilweise auch biotischen Rohstoffen fokussiert. Häufig ist von Materialeffizienz die Rede, beispielsweise bei der Deutschen Materialeffizienzagentur (DEMEA). Doch warum ist Ressourceneffizienz oder Materialeffizienz von Bedeutung?

\section{Ressourcen als Kostenfaktor}

Erstens stellt der Materialeinsatz für Unternehmen schlicht einen Faktoreneinsatz dar, der mit Kosten verbunden ist. Eine effiziente Verwendung ist die Umsetzung des ökonomischen
Prinzips, und sie lässt sich sogar in Euros ausdrücken. Trotzdem ist das Einsparpotenzial bei Unternehmen überraschend groß. Etwa 45 Prozent der Kosten fallen bei einem durchschnittlichen deutschen Unternehmen der produzierenden Wirtschaft im Einsatz von Rohstoffen, Hilfs- und Betriebsstoffen, Werkstoffen oder Vorprodukten an. Zum Vergleich: Für Energie werden nur etwa zwei bis drei Prozent der Kosten aufgewendet.

Die wahren ökonomischen Einsparpotenziale liegen deshalb nicht bei der Beleuchtung, der Abwärme oder der Druckluft, sondern bei den Werkstoffen, ihrer sparsamen Verwendung und der Vermeidung von Reststoffen (Schmidt/Schneider 2010). Je nach Studie werden sie auf fünf bis 15 Prozent der Materialkosten beziffert; bei 45 Prozent Kostenanteil ist das ein beträchtlicher Betrag (Schmidt/Schneider 2013). In diesen Materialströmen liegen auch die größeren Energieeinsparpotenziale der Produktion, denn mit verringertem Materialeinsatz werden auch Energie und Umweltbelastungen für die Bereitstellung der Rohstoffe eingespart. Diese Einsparungen werden in monetären Werten ausgedrückt - denn der Adressat ist der Akteur im Unternehmen. Mit der Kostensenkung verbunden ist aber auch eine Verringerung der physischen Mengen, was aus ökologischen Gründen durchaus sinnvoll ist.

Als zweiter Punkt kann angeführt werden, was im Rahmen der Diskussionen zu der Richtlinie 4800 des Vereins Deutscher Ingenieure (VDI) zum Thema Ressourceneffizienz herausgearbeitet wurde: Übergeordnetes Ziel ist die Schonung natürlicher Ressourcen und die Minderung der Umweltbelastungen. Bei der Ressourceneffizienz wird dem mengenmäßigen oder ökologischen Aufwand stets ein Ertrag oder Nutzen gegenübergestellt - ganz im Sinne des Effizienzansatzes. Nicht thematisiert wird also das absolute Nutzenniveau, etwa im Sinne von Suffizienzstrategien.

\section{Umweltschutz durch Ressourcenschonung}

Man kann an dieser Stelle fragen, ob der Nichtabbau von natürlichen Ressourcen bereits ein eigenes Schutzziel ist oder ob es nicht allein die Umweltbelastungen, der Energieeinsatz und die Landschafts- und Natureingriffe sind, die mit der Nutzung der natürlichen Ressourcen verbunden sind und die es zu verringern gilt. Wäre dem so, wäre eine Ressourceneffizienzanalyse nichts anderes als eine Ökobilanz (Life Cycle Assessment) und man würde vielleicht besser den Energieeinsatz oder die Kohlenstoffdioxid-Emissionen anstatt die Menge abiotischer Rohstoffe quantifizieren. 
Diskussionen wie jüngst auf dem 55. Life Cycle Forum an der Eidgenössischen Technischen Hochschule in Zürich über die abiotischen Ressourcen zeigen allerdings in die Richtung, dass die Entnahme endlicher Ressourcen aus der Natur als solche gezählt werden soll. Man könnte das als die konsequente Anwendung der intergenerationellen Gerechtigkeit innerhalb einer Nachhaltigkeitsstrategie interpretieren: Was heute weniger genutzt wird, steht zukünftigen Generationen noch zur Verfügung.

Darin liegt auch begründet, dass die Ressourcen nun in physischen Mengen, also in Tonnen, angegeben werden müssen, so wie sie der Natur entnommen werden. Das könnte beispielsweise ein Kumulierter Rohstoffaufwand (KRA) sein - analog zum Kumulierten Energieaufwand (KEA) nach der VDI-Richtlinie 4600. Zwei Aspekte sind hier allerdings von Bedeutung: Zum einen bedingt ein solcher Ansatz, dass man dann den Ressourceneinsatz auch zurückverfolgt bis zur Entnahme aus der Natur. Der entsprechende Indikator aus der Nachhaltigkeitsstrategie der Bundesregierung, die Rohstoffproduktivität, enthält bisher nur die inländische Entnahme von Rohstoffen sowie den Import von Gütern, quasi ab Grenzübertritt. Berücksichtigt werden muss aber auch der Rohstoffeinsatz der importierten Güter. Damit wird eine Art Lebenswegbetrachtung zwingend notwendig. Der Rohstoffeinsatz müsste also bis zur Mine zurückverfolgt werden. Die geplante VDI-Richtlinie 4800 verlangt genau einen solchen Ansatz.

Zum anderen müsste man die Ressourcen nach ihrer Art und Qualität differenzieren. Eine Tonne Kies ist nicht das Gleiche wie eine Tonne Tantal, Seltene Erden oder Gold. Das Problem liegt weniger darin, ob man die einzelnen Rohstoffe explizit benennt oder sie entsprechend dem Materialinput pro Serviceeinheit-Ansatz (MIPS) des Wuppertal-Instituts mit der Menge bewegten Materials bewertet und aggregiert. Vielmehr liegen die erforderlichen Daten über die einzelnen Rohstoffe in der Praxis kaum vor. Selten kann ein Unternehmen benennen, welche Spurenstoffe in den eingekauften Vorprodukten enthalten sind. Entsprechende Analysen werden damit zu einer aufwendigen Detektivarbeit.

\section{Ressourcenabhängigkeit als Risiko}

Damit ist auch der dritte Punkt, warum Ressourceneffizienz relevant ist, aufgerufen: die Knappheit einzelner Rohstoffe. Interessanterweise steht hier nicht mehr die geologische Knappheit bestimmter chemischer Elemente oder Mineralien im Vordergrund, wie dies noch vor 40 Jahren vom Club of Rome thematisiert wurde. Gemeint ist die ökonomische Knappheit, also das Versorgungsrisiko eines Wirtschaftssystems, das bei der Herstellung seiner Hightechprodukte inzwischen auf viele spezielle Rohstoffe angewiesen ist: Tantal, Indium, Neodym, Dysprosium und viele andere mehr.

Die Versorgung kann durch politische, soziale oder ökonomische Entwicklungen gefährdet sein, auch durch Naturkatastrophen. Dazu kommt die Verletzlichkeit und Abhängig- keit einzelner Betriebe oder ganzer Volkswirtschaften von den jeweiligen Rohstoffen. In der Fachdiskussion wird dies unter dem Begriff der Rohstoffkritikalität zusammengefasst.

Auf Rohstoffkritikalität kann durch viele Maßnahmen reagiert werden: Ersatz von kritischen Rohstoffen durch weniger kritische Materialien, Produktoptimierung mit geringem Mengeneinsatz oder Rückgewinnung von kritischen Rohstoffen durch Kreislaufsysteme. Teilweise werden damit auch ökologische Ziele verfolgt, doch hinter dem Kritikalitätsansatz an sich steht ein rein wirtschaftliches Ziel. Doch das Thema der Versorgungssicherheit durch kritische Rohstoffe war der hauptsächliche Anlass für viele Industriebetriebe und Wirtschaftsverbände, über die Ressourceneffizienz überhaupt nachzudenken. Auch im Handeln der Bundesregierung spielt die Sicherung der Rohstoffversorgung die eigentlich zentrale Rolle, weniger die ökologischen oder nachhaltigen Aspekte.

\section{Schnittpunkte zur Industrial Ecology}

Wie muss das nun im Kontext einer Industrial Ecology bewertet werden? In der Diskussion über Ressourceneffizienz fühlen sich viele Akteure - aus unterschiedlichen Gründen angesprochen. Gemeinsam setzen sie sich mit dem Stoffmetabolismus eines komplexen Systems auseinander, mit den ökologischen, den ökonomischen, auch den sozialen oder politischen Implikationen.

Die Beweggründe mögen unterschiedlich sein, bei vielen Forderungen und Handlungen treffen sich aber die Interessen, etwa wenn es um die Stärkung des Kreislaufgedankens oder um Alternativen im Produktdesign geht. Das sollte man als eine Chance für einen spannenden Diskurs zwischen Praxis und Theorie, zwischen Wirtschaft und Ökologie begreifen, ohne die Augen davor zu verschließen, dass es auch Widersprüche in den Zielsetzungen und in den Maßnahmen geben kann. Besonders die Frage nach den Möglichkeiten und Grenzen von Effizienzstrategien dürfte hier herausstechen - in vielerlei Hinsicht.

\section{Literatur}

Schmidt, M./Schneider, M. (2010): Kosteneinsparung durch Ressourceneffizienz in produzierenden Unternehmen. Umweltwirtschaftsforum 18/3-4, S. 153-164.

Schmidt, M./Schneider, M. (2013): Ressourceneffizienz spart Produktionskosten. In: Rohn, H./Pastewski, N./Lettenmeier, M. (Hrsg.): Ressourceneffizienz. Stuttgart, S. 9-19.

AUTOR + KONTAKT

Dr. Mario Schmidt ist Professor für ökologische Unternehmensführung an der Hochschule Pforzheim.

Hochschule Pforzheim, Tiefenbronner Str. 65, 75175 Pforzheim. E-Mail: mario.schmidt@hs-pforzheim.de

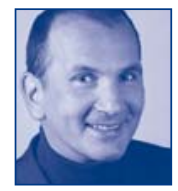

